\title{
Puerarin promotes DUSP1 expression by regulating miR-133a-3p in breast cancer
}

\author{
ZHIFENG LI ${ }^{1}$, WEIWEI XU ${ }^{2}$, XIAOYAN REN ${ }^{3}$, JINHUA XU $^{4}$ and JIANXIN CHEN ${ }^{1}$ \\ ${ }^{1}$ Department of Breast Surgery, Nantong Maternity and Child Health Care Hospital Affiliated to Nantong University; \\ ${ }^{2}$ Department of Oncology, Nantong Tumour Hospital Affiliated to Nantong University; Departments of ${ }^{3}$ Pathology and \\ ${ }^{4}$ Traditional Chinese Medicine, Nantong Maternity and Child Health Care Hospital \\ Affiliated to Nantong University, Nantong, Jiangsu 226001, P.R. China
}

Received March 23, 2018; Accepted October 23, 2018

DOI: $10.3892 / \mathrm{mmr} .2018 .9682$

\begin{abstract}
Previous studies demonstrated that puerarin represents a potential therapeutic drug for breast cancer treatment, due to its ability to inhibit the migration of MCF-7 and MDA-MB-231 cell lines. In order to investigate the mechanism of puerarin in breast cancer cells, the aim of the present study was to examine whether puerarin regulated the dual specificity phosphatase 1 (DUSP1) expression level by promoting the microRNA-133a-3p (miR-133a-3p) expression level in breast cancer. Cell viability and apoptosis were assessed in HCC 38 cells by Cell Counting Kit- 8 assays and a flow cytometry assay, respectively. In total, four treatment groups were considered: Puerarin treatment, miR-133a-3p mimics transfection, puerarin + miR-133a-3p mimics and negative control. miR-133a-3p expression and DUSP1 mRNA expression levels were analyzed by reverse transcription-quantitative polymerase chain reaction, and western blotting was used to detect the protein expression level. Furthermore, a luciferase reporter gene assay was used to test whether DUSP1 mRNA was a direct target of miR-133a-3p. The present results suggested that treatment with puerarin or miR-133a-3p mimics transfection affected the miR-133a-3p expression level and the activity of the DUSP1/p38 pathway, leading to inhibition of HCC38 cell viability and an increase in apoptosis. miR-133a-3p overexpression enhanced the drug action of peurarin. In conclusion, puerarin may increase DUSP1 expression by promoting the miR-133a-3p expression level in HCC38 breast cancer cells. Therefore, miR-133a-3p may represent a novel molecular marker for diagnosis and treatment of breast
\end{abstract}

Correspondence to: Professor Jianxin Chen, Department of Breast Surgery, Nantong Maternity and Child Health Care Hospital Affiliated to Nantong University, 399 Century Avenue, Nantong, Jiangsu 226001, P.R. China

E-mail: chenjianxinjsnt@163.com

Key words: puerarin, breast cancer, dual specificity phosphatase 1, microRNA-133a-3p cancer, and puerarin may represent a promising clinical drug for treatment of patients with breast cancer.

\section{Introduction}

Breast cancer is one of the most common types of malignant tumor in women. Previous studies demonstrated that the rising incidence of breast cancer in Asian women is primarily due to modifications of reproductive and lifestyle factors, including reduced fertility, advancing age of the first pregnancy, decrease in breastfeeding and increase of body mass index $(1,2)$. Furthermore, the age of the first pregnancy, late-onset menopause, height and higher education level were positively associated with high mammographic density (MD) (3). In contrast, age, positive family history of breast cancer and longer durations of breastfeeding were negatively associated with MD among Chinese women with an increased risk of breast cancer (3). MicroRNAs (miRNAs) are a group of short non-coding RNAs (4). A previous study demonstrated that miRNAs were involved in the growth and development of breast cancer, and may be used as biomarkers for cancer diagnosis and prognosis (5). Therefore, it was suggested that miRNAs may represent promising targets for treatment of cancer.

miRNA-133a-3p (miR-133a-3p) is a member of the miRNA family, and previous studies demonstrated that miR-133a-3p was downregulated in multiple types of cancer, including prostate cancer, colorectal cancer, ovarian cancer and breast cancer, and that the downregulation of miR-133a-3p may lead to tumor growth, inhibiting apoptosis in cancer cells (6-8). A previous bioinformatics study identified that miR-133a-3p possessed great diagnostic potential and its decreased expression level suggested that it may represent a novel biomarker for bladder cancer (9). Furthermore, a previous study suggested that miR-133a-3p modulated the expression of SUMO-specific protease 1 (SENP1) by binding to its 3'-untranslated region (3'UTR), resulting in its downregulation (10). Additionally, miR-133a-3p served a role in colorectal cancer (10). In the present study, it was identified that miR-133a-3p may bind to the 3'UTR of dual specificity phosphatase 1 (DUSP1) mRNA using bioinformatics analysis. Overexpression of miR-133a-3p led to a significant increase 
in the DUSP1 expression level. Chen et al (11) demonstrated that progesterone receptors inhibited the proliferation of breast cancer cells by inducing DUSP1 expression. Therefore, the present study suggested that miR-133a-3p may increase the DUSP1 expression level, inducing DUSP1 downstream genes, serving a role in the regulation of cancer growth.

Puerarin is the principal bioactive agent isolated from the root of Pueraria lobata (Willd.) Ohwi, which is additionally termed Gegen in traditional Chinese medicine (12). Puerarin was involved in the regulation of certain molecular targets, including miR-16, miR-214 and miR-106b (13-15), modulating cancer cell proliferation, apoptosis and the cell cycle. Nonetheless, the effect of puerarin on DUSP1 regulation remains to be investigated. The present study identified a novel association between puerarin and DUSP1; the effect of puerarin on breast cancer cells and its underlying mechanism was investigated by bioinformatics and cytological analyses.

\section{Materials and methods}

Cell culture. The human breast cancer cell HCC38 (Shanghai Institute of Cell Biology, Shanghai, China) was cultured in Dulbecco's modified Eagle's medium (Gibco; Thermo Fisher Scientific, Inc., Waltham, MA, USA) with $10 \%$ fetal bovine serum (FBS; Gibco; Thermo Fisher Scientific, Inc.), 100 mg/ml streptomycin and $100 \mathrm{U} / \mathrm{ml}$ penicillin (Beyotime Institute of Biotechnology, Haimen, China) at $37^{\circ} \mathrm{C}$ with $5 \% \mathrm{CO}_{2}$.

Cell transfection. In total, $5 \mu 1$ Lipofectamine ${ }^{\circledR} 2000$ (Invitrogen; Thermo Fisher Scientific, Inc.) solution was used for the transfection of miRNA mimics and inhibitor of $20 \mu \mathrm{M}$ : miR-133a-3p mimics, 5'-UUUGGUCCCCUUCAA CCAGCUG-3'; miR-133a-3p inhibitor, 5'-GCUGGUUGA AGGGGACCAAAUU-3'. After $24 \mathrm{~h}$ of cell transfection, follow-up experiments were carried out. HCC 38 cells were divided into four groups: Cells treated with $20 \mu \mathrm{M}$ puerarin (National Institutes for Food and Drug Control, Beijing, China) group, miR-133a-3p mimics (Shanghai GenePharma Co., Ltd., Shanghai, China) transfection group, $20 \mu \mathrm{M}$ puerarin + miR-133a-3p mimics transfection group and negative control $(20 \mu \mathrm{M}$ puerarin + miR-133a-3p mimics control $)$ transfection group. Cells were transfected at $55-65 \%$ confluence. Cells were incubated under the conditions of $5 \% \mathrm{CO}_{2}$ atmosphere at $37^{\circ} \mathrm{C}$.

Reverse transcription-quantitative polymerase chain reaction (RT-qPCR). HCC38 cells were treated with 0, 10, 20 and $40 \mu \mathrm{M}$ puerarin (16) in $5 \% \mathrm{CO}_{2}$ atmosphere at $37^{\circ} \mathrm{C}$ for $24 \mathrm{~h}$. The total RNA was extracted from the four groups of cells using TRIzol ${ }^{\circledR}$ reagent (Thermo Fisher Scientific, Inc.) according to the manufacturer's protocol. A PrimeScript ${ }^{\mathrm{TM}}$ RT reagent kit (Takara Bio, Inc., Otsu, Japan) was used to synthesize the cDNA $\left(37^{\circ} \mathrm{C}\right.$ for $15 \mathrm{~min} ; 85^{\circ} \mathrm{C}$ for $\left.5 \mathrm{sec}\right)$. The primer sequences are listed in Table I. RT-qPCR was performed using SYBR Premix Ex Taq (Takara Bio, Inc.) and the signal was detected using the ABI-7500 system (Applied Biosystems; Thermo Fisher Scientific, Inc.) according to the manufacturer's protocol: $95^{\circ} \mathrm{C}$ for $30 \mathrm{sec}$, then $95^{\circ} \mathrm{C}$ for $5 \mathrm{sec}, 56^{\circ} \mathrm{C}$ for $20 \mathrm{sec}$ and $72^{\circ} \mathrm{C}$ for $30 \mathrm{sec}$ for 40 cycles. Each RT-qPCR was repeated three times. miR-133a-3p and DUSP1 mRNA relative expression levels were measured using the $2^{-\Delta \Delta \mathrm{Cq}}$ method and GAPDH was used as reference gene (17).

Western blotting. The proteins from the four groups of HCC38 cells were extracted using radioimmunoprecipitation lysis buffer (Beyotime Institute of Biotechnology) and the protein concentration was measured using a Bicinchoninic Acid Protein Assay kit (Beyotime Institute of Biotechnology). The proteins were loaded at $20 \mu \mathrm{g} / \mathrm{lane}$ and separated by $10 \%$ SDS-PAGE. Subsequently, proteins were transferred onto polyvinylidene difluoride membranes (Bio-Rad Laboratories, Inc., Hercules, CA, USA). In total, 0.1\% TBST was used to block the transfer membranes, which were subsequently incubated with $5 \%$ non-fat dry milk at $25^{\circ} \mathrm{C}$ for $1 \mathrm{~h}$, and the primary antibodies were incubated at $4^{\circ} \mathrm{C}$ overnight, and second antibody were incubated for $1 \mathrm{~h}$ at room temperature. Densitometric analysis by DAB visualization reagent (Beyotime Institute of Biotechnology) was performed to detect protein expression level using ImageJ software (version 1.49; National Institutes of Health, Bethesda, MD, USA). The antibodies for cleaved caspase-9 (cat. no. 9505, 1:1,000), B-cell lymphoma 2 (Bcl-2; cat. no. 4223, 1:1,000), Bcl2 associated X (Bax; cat. no. 5023, 1:1,000), p38 (cat. no. 8690, 1:1,000), phosphorylated p38 (p-p38; cat. no. 4511, 1:1,000) and $\beta$-actin (cat. no. 3700, 1:1,000) were purchased from Cell Signaling Technology, Inc. (Danvers, MA, USA), and DUSP1 antibody (cat. no. ab61201, 1:1,000 ) was purchased from Abcam (Cambridge, UK), and the secondary antibody (rabbit/mouse horseradish peroxidase-conjugated anti-goat immunoglobulin G, (cat. no. A0208; 1:1,000) was purchased from Beyotime Institute of Biotechnology.

Cell viability assay. Cell viability was detected by a Cell Counting Kit-8 (CCK-8; Dojindo Molecular Technologies, Inc., Kumamoto, Japan). HCC38 cells were cultured in $90 \mu 1$ complete medium, in 96-well plates, $2 \times 10^{4}$ cells/well. Triplicate repetitions wells were used for each group. Cells were incubated for $8 \mathrm{~h}$ and subsequently treated with various doses of puerarin for 24,48 and $72 \mathrm{~h}$ in 96-well plates at $37^{\circ} \mathrm{C}$. Subsequently, $10 \mu 1 \mathrm{CCK}-8$ solution and $90 \mu 1 \mathrm{FBS}-$ free medium were mixed, and added to each well for incubation for $10 \mathrm{~min}$ at $37^{\circ} \mathrm{C}$. The absorbance was detected at $450 \mathrm{~nm}$ using a microplate reader (BioTek Instruments, Inc., Winooski, VT, USA) to measure cell viability.

Cell apoptosis analysis by flow cytometry (FCM). HCC 38 cell apoptosis was measured in the four groups by FCM, using an Annexin V-fluorescein isothiocyanate (V-FITC) apoptosis detection kit (Beyotime Institute of Biotechnology). HCC38 cells were cultured, collected and washed with cold PBS twice, and subsequently treated with puerarin for $48 \mathrm{~h}$ at $37^{\circ} \mathrm{C}$. Cells were treated with Annexin propidium iodide and V-FITC. Subsequently the cells were centrifuged at $100 \mathrm{xg}$ at $25^{\circ} \mathrm{C}$ for $5 \mathrm{~min}$. Cell apoptosis was measured using a flow cytometer and FlowJo software (version 10; FlowJo LLC, Ashland, OR, USA).

Caspase- 3 activity analysis. Caspase- 3 activity, measured to detect cell apoptosis, was assessed using a caspase-3 colorimetric activity assay kit (Beyotime Institute of Biotechnology) 
Table I. Primer sequences used for polymerase chain reaction.

\begin{tabular}{lll}
\hline Gene & \multicolumn{1}{c}{ Sense primer $\left(5^{\prime} \rightarrow 3^{\prime}\right)$} & Antisense primer $\left(5^{\prime} \rightarrow 3^{\prime}\right)$ \\
\hline DUSP1 & TCGCTGCGAAGGACATTTGGG & AGCGACGCTTCCTGTAAACCC \\
miR-133a-3p & GGGAGCCAAATGCTTTGCTAG & CCCTCGGTTTACGAAACGATC \\
GAPDH & CGGAGTCAACGGATTTGGTCGTAT & AGCCTTCTCCATGGTGGTGAAGAC \\
U6 & TGCGGGTGCTCGCTTCGGCAGC & CCAGTGCAGGGTCCGAGGT
\end{tabular}

DUSP1, dual specificity phosphatase 1 ; miR, microRNA.

according to the manufacturer's protocol. Cells were collected and washed twice with coldPBS, and the cells were subsequently lysed with the buffer provided in the kit. The caspase-3 substrate N-Acetyl-Asp-Glu-Val-Asp-7-amido-4-Methylcoumarin was used to incubate an equal amount of protein $(100 \mu \mathrm{g})$ at $37^{\circ} \mathrm{C}$ for $2 \mathrm{~h}$. Caspase-3 activity was measured based on the absorbance at $405 \mathrm{~nm}$ using a plate reader.

Luciferase reporter assay. The interaction between miR-133a-3p and the 3'UTR of DUSP1 was predicted by TargetScanHuman version 7.1 (www.targetscan.org/vert_71/). The target sequence was cloned in pcDNA3.1- Luciferase vector (Promega Corporation, Madison, WI, USA) with firefly and Renilla luciferase using XbaI. In total, two plasmids were generated: DUSP1 wild-type (DUSP1-WT) containing the 3'UTR of DUSP1 and the predicted binding site, and DUSP1-mutant (DUSP1-MT) containing a mutated binding site in the 3'UTR. The mimics and vectors were co-transfected into HCC38 cells using Lipofectamine ${ }^{\circledR} 2000$ (Invitrogen; Thermo Fisher Scientific, Inc.) at $37^{\circ} \mathrm{C}$ for $48 \mathrm{~h}$. Subsequently, the transfected HCC 38 cells were analyzed using a dual-luciferase reporter assay system (Promega Corporation). Each experiment was replicated three times independently. Firefly luciferase activity was normalized to Renilla luciferase activity.

Statistical analysis. Data are presented as the mean \pm standard error. Each experiment was replicated three times independently. All the data were analyzed using SPSS (17.0; SPSS, Inc., Chicago, IL, USA). Student's t-test or one-way analysis of variance followed by Student-Newman-Keuls test was used to analyze the differences between two or multiple groups, respectively. $\mathrm{P}<0.05$ was considered to indicate a statistically significant difference.

\section{Results}

miR-133a-3p expression level is upregulated by puerarin and miR-133a-3p mimics. Previous studies identified that puerarin inhibited the development of tumors, and miR-133a-3p served a role in suppressing cancer (18). The aim of the present study was to investigate the effect of puerarin on the miR-133a-3p expression level in breast cancer. The present results demonstrated that the miR-133a-3p expression level was increased upon treatment with puerarin in HCC38 cells in a dose-dependent manner, suggesting that puerarin may possess antitumor activity (Fig. 1A). The concentration of $20 \mu \mathrm{M}$ puerarin
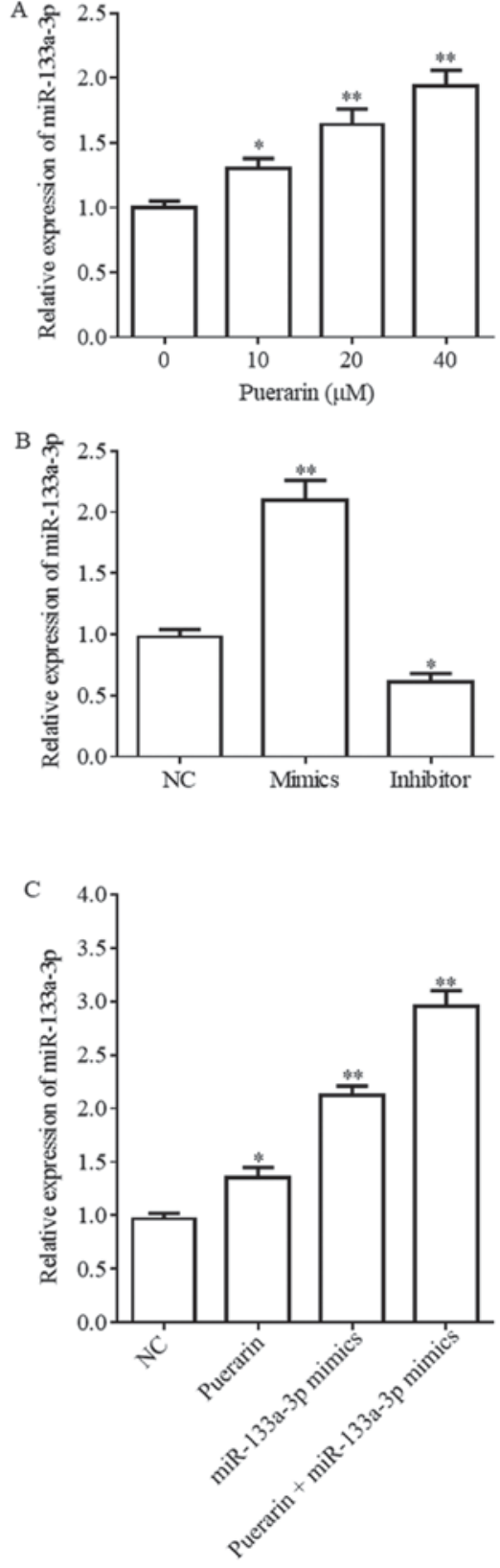

Figure 1. Puerarin and miR-133a-3p mimics promote the miR-133a-3p expression level. (A) HCC38 cells treated with puerarin $(0,10,20$ and $40 \mu \mathrm{M})$. Expression level of miR-133a-3p detected by RT-qPCR. (B) miR-133a-3p expression level is affected by miR-133a-3p inhibitor and miR-133a-3p mimics. (C) Expression level of miR-133a-3p detected by RT-qPCR in HCC38 treated with $20 \mu \mathrm{M}$ puerarin, miR-133a-3p mimics, $20 \mu \mathrm{M}$ puerarin + miR-133a-3p mimics and NC group. ${ }^{*} \mathrm{P}<0.05,{ }^{* *} \mathrm{P}<0.01$ vs. respective NC. NC, negative control; RT-qPCR, reverse transcription-quantitative polymerase chain reaction; miR, microRNA. 

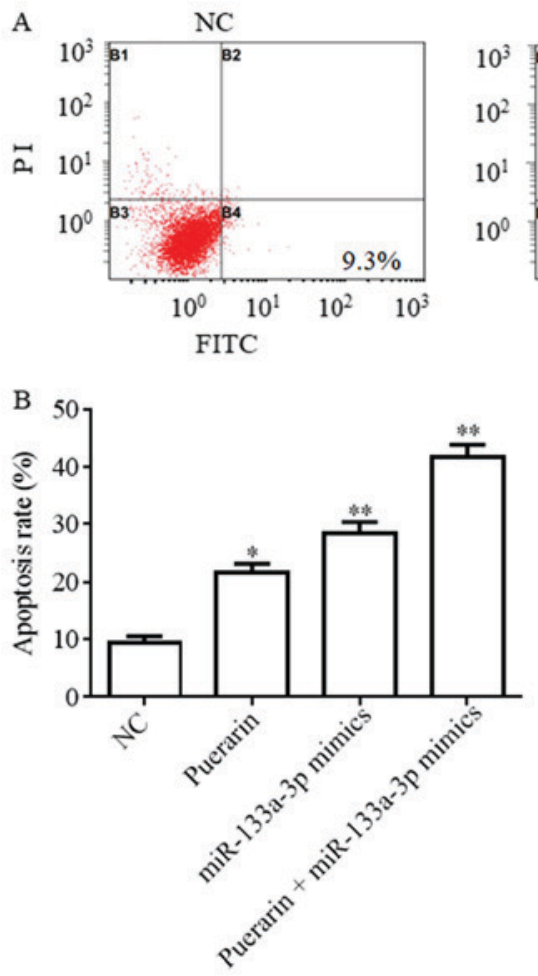
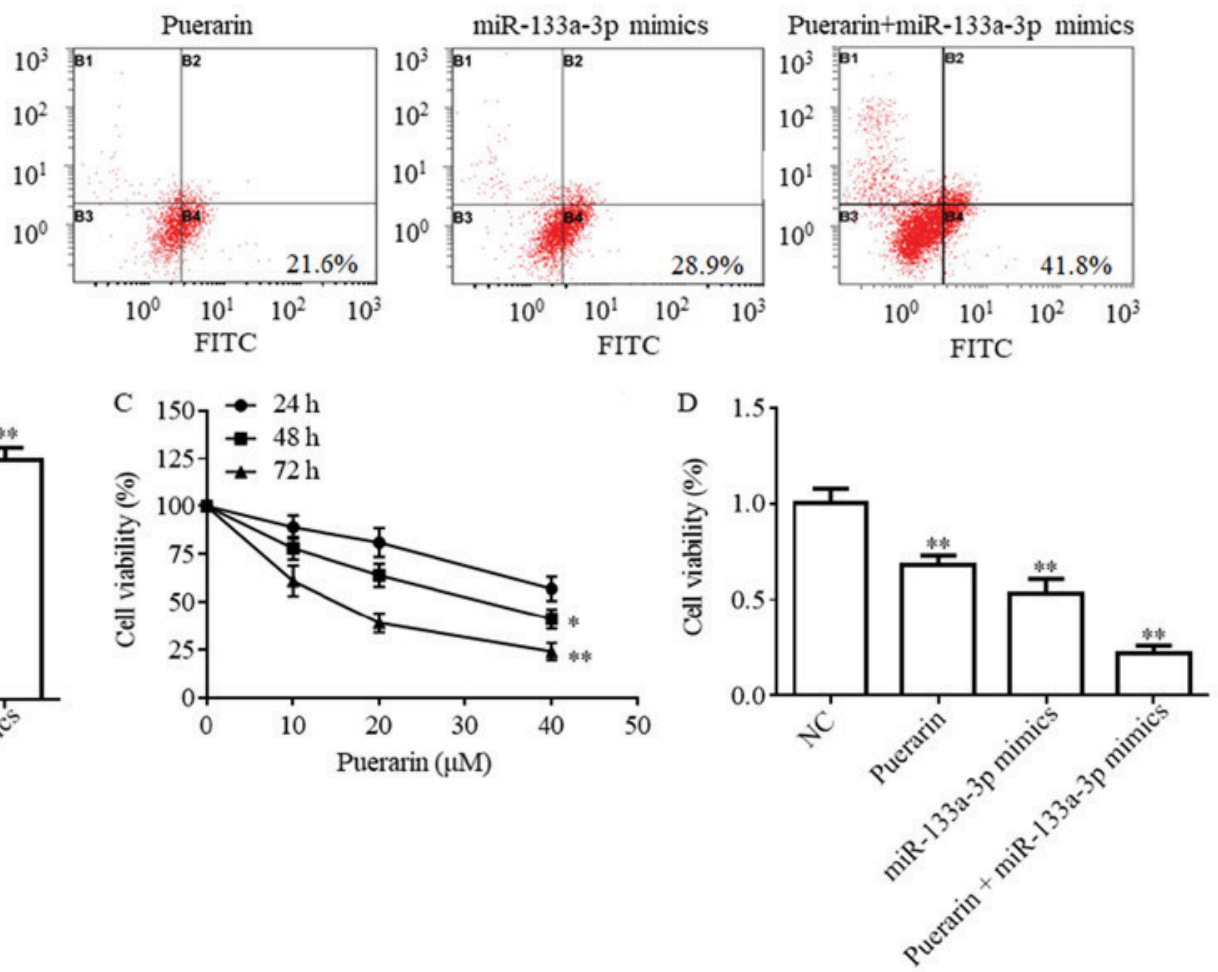

Figure 2. Puerarin promotes cell apoptosis and inhibits cell viability of breast cancer. (A) HCC38 cell apoptotic rate following treatment with $20 \mu \mathrm{M}$ puerarin miR-133a-3p mimics, $20 \mu \mathrm{M}$ puerarin + miR-133a-3p mimics and NC group detected by a flow cytometry assay. ${ }^{*} \mathrm{P}<0.05,{ }^{* *} \mathrm{P}<0.01 \mathrm{vs}$. NC. (B) Apoptosis rate expressed as a percentage. (C) Cell viability following the treatment with puerarin at various concentrations at 24,48 and 72 h, detected by a CCK- 8 assay. ${ }^{*} \mathrm{P}<0.05,{ }^{* *} \mathrm{P}<0.01$ vs. $24 \mathrm{~h}$. (D) Cell viability detected using a CCK-8 assay in four treatment groups. ${ }^{* *} \mathrm{P}<0.01$ vs. NC. NC, negative control; CCK-8, Cell Counting Kit-8; miR, microRNA; PI, propidium iodide; FITC, fluorescein isothiocyanate.

demonstrated the best experimental results. The effect of miR-133a-3p mimics and inhibitor is shown in Fig. 1B; the miR-133a-3p expression level was increased following treatment with puerarin $(20 \mu \mathrm{M}), \operatorname{miR}-133 \mathrm{a}-3 \mathrm{p}$ mimics and puerarin $(20 \mu \mathrm{M})+$ miR-133a-3p mimics compared with the NC group (Fig. 1C).

Puerarin and miR-133a-3p mimics inhibit cell viability and promotes cell apoptosis. A CCK-8 assay was conducted to test the effect of puerarin on the viability of breast cancer cells. Flow cytometry results suggested that the apoptotic rates following treatment with puerarin $(20 \mu \mathrm{M})$, miR-133a-3p mimics and puerarin $(20 \mu \mathrm{M})+\operatorname{miR}-133 \mathrm{a}-3 \mathrm{p}$ mimics were significantly increased compared with the NC group (Fig. 2A and $\mathrm{B} ; \mathrm{P}<0.05$ ). Furthermore, the apoptotic rate of the puerarin $(20 \mu \mathrm{M})+$ miR-133a-3p mimics group was increased compared with the puerarin and miR-133a-3p mimics groups (Fig. 2A and B). The CCK-8 assay suggested that puerarin inhibited the viability of HCC 38 cells in a timeand dose-dependent manner (Fig. 2C). Furthermore, the cell viability in the miR-133a-3p mimics, puerarin and puerarin + miR-133a-3p mimics groups was significantly decreased compared with the NC group (Fig. 2D; $\mathrm{P}<0.01$ ).

Protein expression levels of apoptosis-associated proteins are regulated by puerarin and miR $-133 a-3 p$ mimics. The flow cytometry results suggested that puerarin and miR-133a-3p mimics promoted HCC38 cell apoptosis. Therefore, the expression levels of proteins associated with apoptosis and caspase-3 activity were investigated (Fig. 3). The western blotting assay suggested that Bax and cleaved caspase-9 expression levels were significantly increased in the puerarin $(20 \mu \mathrm{M})$, miR-133a-3p mimics and puerarin $(20 \mu \mathrm{M})+$ miR-133a-3p mimics groups compared with the NC group; however, Bcl-2 expression exhibited the opposite trend (Fig. 3A and B). Caspase-3 activity was significantly increased following the treatment with puerarin and/or miR-133a-3p mimics (Fig. 3C).

DUSP1 is a direct target of miR-133a-3p. A luciferase reporter gene assay was performed to test whether DUSP1 was a direct target gene of miR-133a-3p. Complementary binding was identified in the sequences of miR-133a-3p and the DUSP1 3'UTR (Fig. 4A). The pcDNA 3.1-Luciferase vector was synthesized and subsequently DUSP1 3'UTR-WT or MT sequences were inserted into the plasmid. The present results demonstrated that the luciferase activity of DUSP1 3'UTR-WT co-transfected with the miR-133a-3p inhibitor was significantly decreased compared with the NC. In addition, no significant difference was detected between the $\mathrm{NC}$ and DUSP1 3'UTR-MT co-transfected with miR-133a-3p inhibitor (Fig. 4B). As miR-133a is positively correlated with DUSP1 expression, inhibitor was used to perform this experiment (Fig. 4C). The expression of DUSP1 protein is also regulated by miR-133a-3p (Fig. 4D). These present results suggested that the sequence of DUSP1 3'UTR was complementary to the miR-133A-3p sequence and that this site may be a direct target of miR-133a-3p. 
A

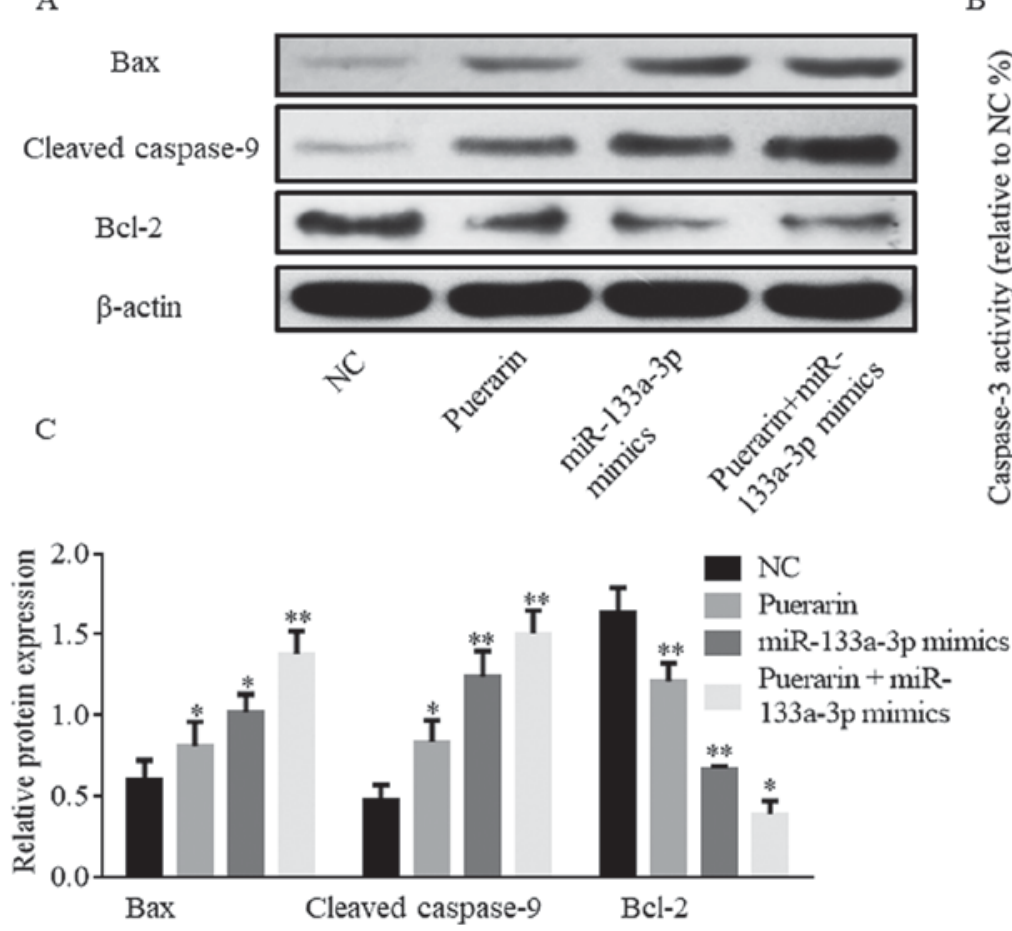

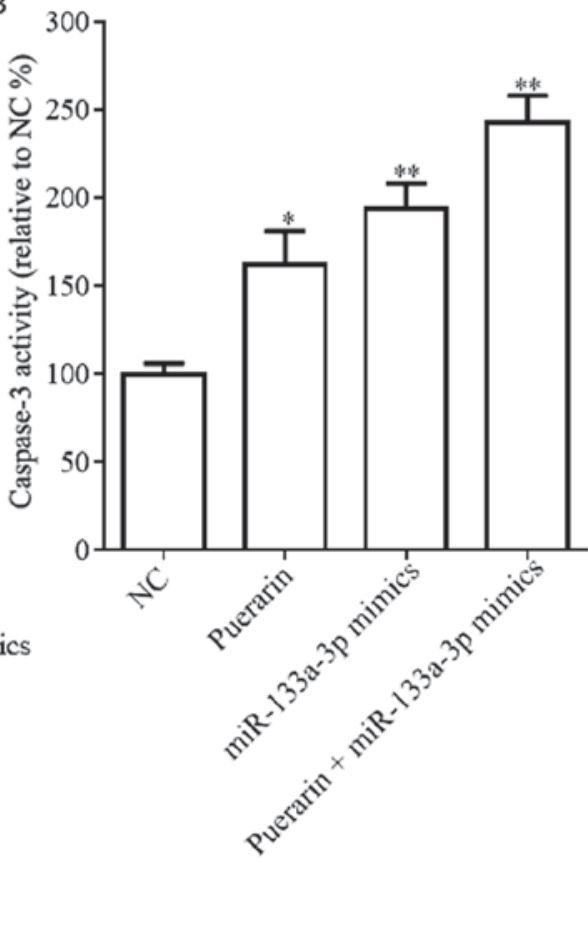

Figure 3. Puerarin and miR-133a-3p mimics regulate Bax, cleaved caspase-9 and Bcl-2 protein expression levels. (A) Protein expression level of Bax, cleaved caspase- 9 and Bcl-2 detected by western blotting. (B) Activity of caspase-3. (C) Densitometric analysis. "P $<0.05,{ }^{* *} \mathrm{P}<0.01$ vs. respective NC. NC, negative control; miR, microRNA; Bcl-2, B-cell lymphoma 2; Bax, Bcl2 associated X. DUSP1 3'-UTR 5, ...GAACUCAGCACAUUCGGGACCAA... DUSP1 3'-UTR mutant ... GAACUCAGCACAUUCGAACGCAA... $\mathrm{C}$

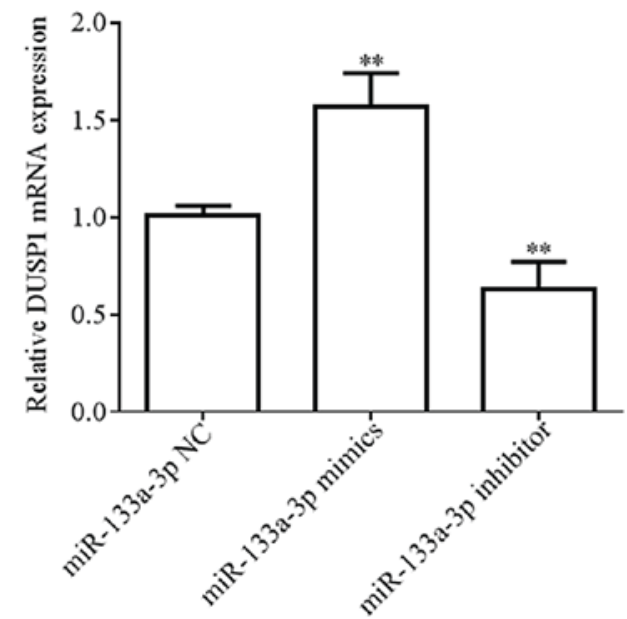

B

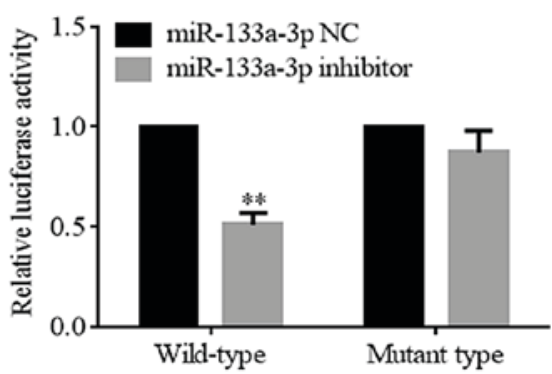

$\mathrm{D}$

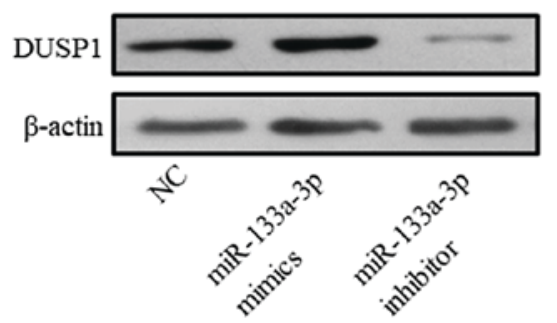

Figure 4. DUSP1 expression level is associated with miR-133a-3p. (A) miR-133a-3p sequence demonstrates complementary binding to the sequence of the 3'UTR of DUSP1. (B) DUSP1 wild-type 3'UTR is associated with miR-133a-3p as observed in the luciferase reporter assay. (C) DUSP1 expression level is positively regulated by miR-133a-3p. (D) Protein expression level of DUSP1 is regulated by miR-133a-3p. ${ }^{* *} \mathrm{P}<0.01 \mathrm{vs}$. NC. NC, negative control; miR, microRNA; DUSP1, dual specificity phosphatase 1; UTR, untranslated region.

Puerarin and miR-133a-3p regulate the DUSP1/p38 mitogen-activated protein kinase (MAPK) pathway. The western blotting assay suggested that puerarin promoted the protein expression level of DUSP1 in a dose-dependent manner
(Fig. 5A). HCC38 cells were transfected with the miR-133a-3p inhibitor and were subsequently analyzed to examine the association between miR-133a-3p and DUSP1 (Fig. 5C and D). The DUSP1 mRNA expression level was increased following 
A

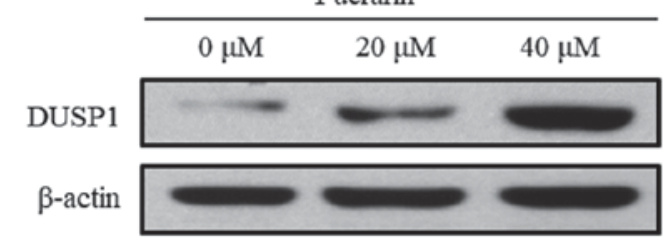

$\mathrm{B}$

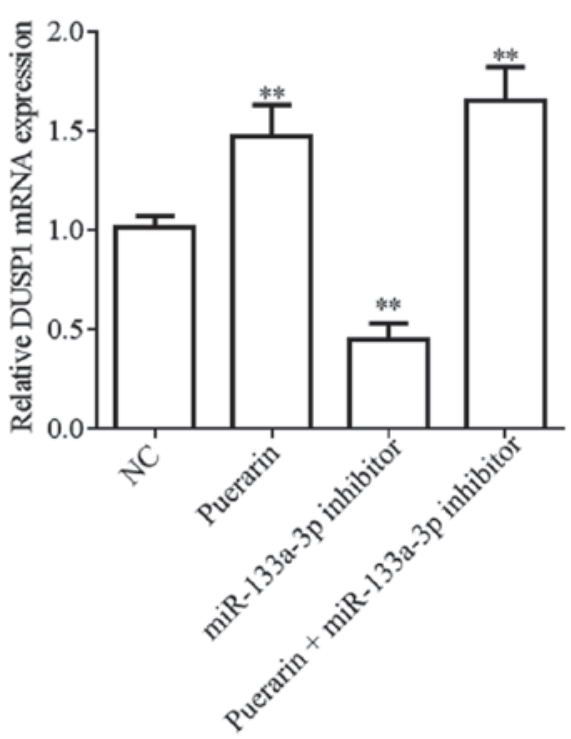

$\mathrm{C}$

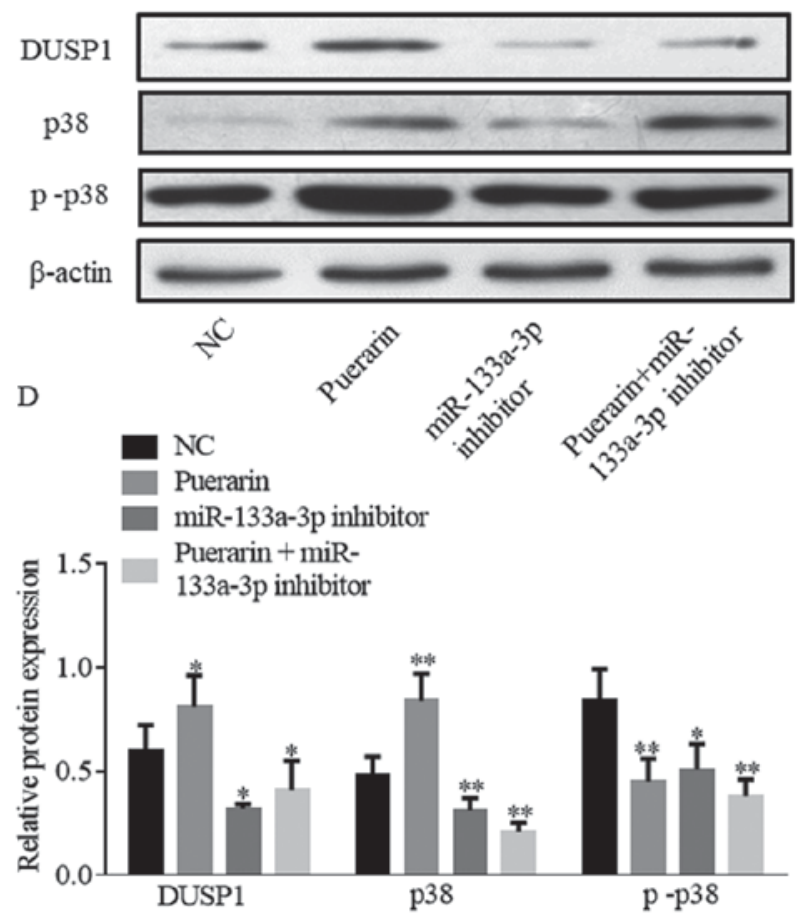

Figure 5. Puerarin promotes the expression of DUSP1 in breast cancer cells. (A) Various concentrations of puerarin increase the DUSP1 protein expression level. (B) Puerarin regulates the expression levels of proteins downstream of DUSP1 by affecting the protein expression level of DUSP1. (C) Puerarin and miR-133a-3p inhibitor regulate the DUSP1 mRNA expression level. (D) Densitometric analysis. " $\mathrm{P}<0.05,{ }^{* *} \mathrm{P}<0.01$ vs. respective NC. NC, negative control; DUSP1, dual specificity phosphatase 1; p-, phosphorylated; miR, microRNA.

treatment with puerarin; however, miR-133a-3p inhibitor led to the opposite effect (Fig. 5B). The protein expression levels of DUSP1 and MAPK were analyzed by western blotting. The present study demonstrated that puerarin significantly increased the DUSP1 and p38 protein expression levels, and decreased the p-p38 expression level (Fig. 5C and D; $\mathrm{P}<0.05$ ). The miR-133a-3p inhibitor attenuated the effect of puerarin (Fig. 5C and D). These present results suggested that DUSP1 may serve tumor suppressive roles via the MAPK pathway.

\section{Discussion}

Breast cancer is the most common invasive cancer among women worldwide, accounting for $16 \%$ of all types of cancer and $22.9 \%$ of the invasive types of cancer in women (19). Numerous previous studies investigated the markers involved in breast cancer cell growth and differentiation, which are essential processes for the formation and development of breast cancer $(20,21)$. Cancer metastasis is the principal cause of the poor prognosis and high mortality of breast cancer (22). At present, the treatment of breast cancer is based on surgery and chemotherapy; however, the emergence of drug resistance limits the efficacy of chemotherapy. The efficacy of traditional Chinese medicine in cancer has been investigated, and numerous Chinese herbs have been demonstrated to inhibit cancer (23). The combination of berberine and evodiamine suppressed the proliferation of MCF-7 cells by inducing cell cycle arrest and apoptosis, suggesting potential synergistic and combinatorial applications of traditional Chinese medicine treatments (24). A previous study, including a total of 1,853 patients with aggressive breast cancer, demonstrated that Chinese herbal products contributed to a decrease in the risk of invasive breast cancer (25). A previous study suggested that puerarin regulated the expression level of proteins involved in the mammalian target of rapamycin/ribosomal protein S6 kinase $\beta-1$ signaling pathway, and suggested that puerarin may represent a novel drug for the treatment of bladder cancer (26). The present study examined whether puerarin had an anticancer effect on breast cancer and investigated its underlying mechanism of action.

At present, anticancer therapy involving miRNAs is a relatively novel field, and miRNA activity and function are principal topics of investigation. Generally, miRNAs may serve as oncogenes or tumor suppressor genes by regulating the target mRNA or protein. SENP1 is one of the targets of miR-133a-3p, and the miR-133a-3p/SENP1 axis was demonstrated to regulate cell proliferation and the cell cycle in colorectal cancer (10). In addition, miR-133a-3p has been identified as a tumor suppressor gene in breast cancer partly by targeting LIM and SH3 domain protein 1 (27). The present study suggested, using a bioinformatics analysis, that the DUSP1 gene may be one of the targets of miR-133a-3p. Therefore, the present study suggested that DUSP1, a gene involved in a number of tumors $(28,29)$, was part of the same pathway of miR-133a-3p in regulating tumorigenesis. Furthermore, a previous study focused on the development of antitumor drugs derived from miRNAs with potential clinical implications (30). A previous study demonstrated that pterostilbene decreased the 
miR-19a expression level, affecting the phosphatase and tensin homolog/RAC- $\alpha$ serine/threonine-protein kinase pathway, inhibiting cell viability, cell cycle arrest and apoptosis, and promoting cell invasion in hepatocellular carcinoma cells (31). In the present study, the effect of puerarin on miR-133a-3p was investigated in breast cancer HCC38 cells. Furthermore, miR-133a-3p was suggested to serve a tumor suppressive role by directly targeting DUSP1. Additionally, the proteins downstream of DUSP1 were investigated.

As puerarin has the potential to suppress the growth of tumor cells, research has focused on understanding the biological activity of puerarin (32). Furthermore, the mechanism of puerarin as an anticancer drug may involve the induction of tumor suppressor genes or the inhibition of oncogenes. The present results suggested that the viability of breast cancer cells HCC38 was suppressed by puerarin. Additionally, apoptosis of HCC38 was promoted, and the present findings provide basis for further investigating the effect of puerarin on breast cancer. In the present study, puerarin promoted the expression of tumor suppressor genes miR-133a-3p and DUSP1, suggesting that puerarin may affect this pathway to serve antitumor effects. Furthermore, according to the bioinformatics analysis, it was identified that miR-133a-3p and DUSP1 genes interacted, based on the nucleotide sequence. The expression of the DUSP1 gene was promoted by the upregulation of miR-133a-3p. Xiao et al (33) demonstrated that miRNAs may promote gene expression by targeting enhancers. DUSPs are a family of proteins that function as negative regulators of MAPK activity in mammalian cells (34). A previous study suggested that silencing DUSP1 promoted the release of pro-inflammatory cytokines by activating the MAPK signaling pathway (35). The present study identified that upregulation of DUSP1 expression led to an increase of the p-p38 MAPK protein expression level.

Collectively, the present study identified that puerarin decreased HCC38 cell viability and promoted apoptosis. The mechanism underlying puerarin involved the upregulation of miR-133a-3p expression. Notably, miR-133a-3p upregulation increased the DUSP1 expression level, causing a decrease in the protein expression level of p-p38. The impact of the downstream genes of the MAPK pathway has not been investigated, representing a limitation of the present study, and additional experiments are required to address this aspect. The present results investigated the molecular mechanisms of puerarin, a drug that may be used in the future for improving the quality of life of the patients with breast cancer.

\section{Acknowledgements}

Not applicable.

\section{Funding}

The present study was supported by The Nature and Science Foundation of Jiangsu Province (China; grant no. 2016032A).

\section{Availability of data and materials}

The datasets used and/or analyzed during the current study are available from the corresponding author on reasonable request.

\section{Authors' contributions}

JC designed the present study. ZL, XR and JX performed the experiments and wrote the paper. WX performed the statistical analysis. All authors read and approved the final manuscript.

\section{Ethics approval and consent to participate}

Not applicable.

\section{Patient consent for publication}

Not applicable.

\section{Competing interests}

The authors declare that they have no competing interests.

\section{References}

1. Chia KS, Reilly M, Tan CS, Lee J, Pawitan Y, Adami HO, Hall P and Mow B: Profound changes in breast cancer incidence may reflect changes into a Westernized lifestyle: A comparative population-based study in Singapore and Sweden. Int J Cancer 113: 302-306, 2005.

2. Leung GM, Thach TQ, Lam TH, Hedley AJ, Foo W, Fielding R, Yip PS, Lau EM and Wong CM: Trends in breast cancer incidence in Hong Kong between 1973 and 1999: An age-period-cohort analysis. Br J Cancer 87: 982-988, 2002.

3. Sung H, Ren J, Li J, Pfeiffer RM, Wang Y, Guida JL, Fang Y, Shi J, Zhang K, Li N, et al: Breast cancer risk factors and mammographic density among high-risk women in urban China. NPJ Breast Cancer 4: 3, 2018.

4. Moss EG: MicroRNAs: Hidden in the genome. Curr Biol 12: R138-R140, 2002.

5. Lv C, Li F, Li X, Tian Y, Zhang Y, Sheng X, Song Y, Meng Q, Yuan S, Luan L, et al: miR-31 promotes mammary stem cell expansion and breast tumorigenesis by suppressing Wnt signaling antagonists. Nat Commun 8: 1036, 2017.

6. Kojima S, Chiyomaru T, Kawakami K, Yoshino H, Enokida H, Nohata N, Fuse M, Ichikawa T, Naya Y, Nakagawa M and Seki N: Tumour suppressors miR-1 and miR-133a target the oncogenic function of purine nucleoside phosphorylase (PNP) in prostate cancer. Br J Cancer 106: 405-413, 2012.

7. Dong Y, Zhao J, Wu CW, Zhang L, Liu X, Kang W, Leung WW, Zhang N, Chan FK, Sung JJ, et al: Tumor suppressor functions of miR-133a in colorectal cancer. Mol Cancer Res 11: 1051-1060, 2013.

8. Guo J, Xia B, Meng F and Lou G: miR-133a suppresses ovarian cancer cell proliferation by directly targeting insulin-like growth factor 1 receptor. Tumour Biol 35: 1557-1564, 2014.

9. Gao L, Li SH, Tian YX, Zhu QQ, Chen G, Pang YY and Hu XH: Role of downregulated miR-133a-3p expression in bladder cancer: A bioinformatics study. OncoTargets Ther 10: 3667-3683, 2017.

10. Zhou GQ, Han F, Shi ZL, Yu L, Li XF, Yu C, Shen CL, Wan DW, Zhu XG, Li R and He SB: miR-133a-3p targets SUMO-specific protease 1 to inhibit cell proliferation and cell cycle progress in colorectal cancer. Oncol Res 26: 795-800, 2018.

11. Chen CC, Hardy DB and Mendelson CR: Progesterone receptor inhibits proliferation of human breast cancer cells via induction of MAPK phosphatase 1 (MKP-1/DUSP1). J Biol Chem 286: 43091-43102, 2011.

12. Zhou YX, Zhang H and Peng C: Puerarin: A review of pharmacological effects. Phytother Res 28: 961-975, 2014.

13. Liu X, Li S, Li Y, Cheng B, Tan B and Wang G: Puerarin inhibits proliferation and induces apoptosis by upregulation of miR-16 in bladder cancer cell line T24. Oncol Res 26: 1227-1234, 2018.

14. Zeng X, Feng Q, Zhao F, Sun C, Zhou T, Yang J and Zhan X: Puerarin inhibits TRPM3/miR-204 to promote MC3T3-E1 cells proliferation, differentiation and mineralization. Phytother Res 32: 996-1003, 2018 
15. Yin M, Yuan Y, Cui Y, Hong X, Luo H, Hu X, Tang M, Hescheler J and Xi J: Puerarin suppresses the self-renewal of murine embryonic stem cells by inhibition of REST-miR-21 regulatory pathway. Cell Physiol Biochem 37: 527-536, 2015.

16. Zhang WG, Liu XF, Meng KW and Hu SY: Puerarin inhibits growth and induces apoptosis in SMMC-7721 hepatocellular carcinoma cells. Mol Med Rep 10: 2752-2758, 2014.

17. Livak KJ and Schmittgen TD: Analysis of relative gene expression data using real-time quantitative PCR and the 2(-Delta Delta C(T)) method. Methods 25: 402-408, 2001.

18. Kang H, Zhang J, Wang B, Liu M, Zhao J, Yang $M$ and Li Y: Puerarin inhibits M2 polarization and metastasis of tumor-associated macrophages from NSCLC xenograft model via inactivating MEK/ERK 1/2 pathway. Int J Oncol 50: 545-554, 2017.

19. Donepudi MS, Kondapalli K, Amos SJ and Venkanteshan P: Breast cancer statistics and markers. J Cancer Res Ther 10: 506-511, 2014

20. Ciriello G, Gatza ML, Beck AH, Wilkerson MD, Rhie SK, Pastore A, Zhang H, McLellan M, Yau C, Kandoth C, et al: Comprehensive molecular portraits of invasive lobular breast cancer. Cell 163: 506-519, 2015.

21. Cancer Genome Atlas Network: Comprehensive molecular portraits of human breast tumours. Nature 490: 61-70, 2012.

22. Alkabban FM and Ferguson T: Cancer, Breast. StatPearls Publishing, Treasure Island, FL, 2018.

23. Xu SY, Huang $X$ and Cheong KL: Recent advances in marine algae polysaccharides: Isolation, structure, and activities. Mar Drugs 15: E388, 2017

24. Du J, Sun Y, Lu YY, Lau E, Zhao M, Zhou QM and Su SB: Berberine and evodiamine act synergistically against human breast cancer MCF-7 cells by inducing cell cycle arrest and apoptosis. Anticancer Res 37: 6141-6151, 2017.

25. Tsai YT, Lai JN, Lo PC, Chen CN and Lin JG: Prescription of Chinese herbal products is associated with a decreased risk of invasive breast cancer. Medicine (Baltimore) 96: e7918, 2017.

26. Jiang K, Chen H, Tang K, Guan W, Zhou H, Guo X, Chen Z, Ye Z and $\mathrm{Xu} \mathrm{H}$ : Puerarin inhibits bladder cancer cell proliferation through the mTOR/p70S6K signaling pathway. Oncol Lett 15: $167-174,2018$.
27. Sui Y, Zhang X, Yang H, Wei W and Wang M: MicroRNA-133a acts as a tumour suppressor in breast cancer through targeting LASP1. Oncol Rep 39: 473-482, 2018

28. Kesarwani M, Kincaid Z, Gomaa A, Huber E, Rohrabaugh S, Siddiqui Z, Bouso MF, Latif T, Xu M, Komurov K, et al: Targeting c-FOS and DUSP1 abrogates intrinsic resistance to tyrosine-kinase inhibitor therapy in BCR-ABL-induced leukemia. Nat Med 23: 472-482, 2017

29. Nettersheim D, Jostes S, Fabry M, Honecker F, Schumacher V, Kirfel J, Kristiansen G and Schorle H: A signaling cascade including ARID1A, GADD45B and DUSP1 induces apoptosis and affects the cell cycle of germ cell cancers after romidepsin treatment. Oncotarget 7: 74931-74946, 2016.

30. Rukov JL and Shomron N: MicroRNA pharmacogenomics: Post-transcriptional regulation of drug response. Trends Mol Med 17: 412-423, 2011.

31. Qian YY, Liu ZS, Zhang Z, Levenson AS and Li K: Pterostilbene increases PTEN expression through the targeted downregulation of microRNA-19a in hepatocellular carcinoma. Mol Med Rep 17: 5193-5201, 2018

32. Wei HY, Zhang YJ and Zhao SZ: Puerarin regulates neovascular glaucoma through pigment epitheliumderived growth factorinduced NFאB signaling pathway. Mol Med Rep 17: 7866-7874, 2018.

33. Xiao M, Li J, Li W, Wang Y, Wu F, Xi Y, Zhang L, Ding C, Luo H, Li Y, et al: MicroRNAs activate gene transcription epigenetically as an enhancer trigger. RNA Biol 14: 1326-1334, 2017.

34. Low HB and Zhang Y: Regulatory roles of MAPK phosphatases in cancer. Immune Netw 16: 85-98, 2016.

35. Zhang B, Li SL, Xie HL, Fan JW, Gu CW, Kang C and Teng MJ Effects of silencing the DUSP1 gene using lentiviral vector-mediated siRNA on the release of proinflammatory cytokines through regulation of the MAPK signaling pathway in mice with acute pancreatitis. Int J Mol Med 41: 2213-2224, 2018.

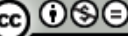

This work is licensed under a Creative Commons Attribution-NonCommercial-NoDerivatives 4.0 International (CC BY-NC-ND 4.0) License. 